\title{
X-linked creatine transporter deficiency
}

INSERM

\section{Source}

INSERM. (1999). Orphanet: an online rare disease and orphan drug data base. $\underline{X \text {-linked }}$ creatine transporter deficiency. ORPHA:52503

X-linked creatine transporter deficiency (CRTR-D) is a creatine deficiency syndrome characterized clinically by global developmental delay/ intellectual disability (DD/ID) with prominent speech/language delay, autistic behavior and seizures. 